\title{
On the efficiency of the method of fundamental solutions for acoustic scattering by a poroelastic material
}

\author{
B. Nennig, E. Perrey-Debain \& J.-D. Chazot \\ Université Technologique de Compiègne, Laboratoire Roberval UMR \\ CNRS 6253, France
}

\begin{abstract}
The Method of Fundamental Solutions is now a well established technique that has proved to be reliable for a specific range of wave problems such as the scattering of acoustic and elastic waves by obstacles and inclusions of regular shapes. The goal of this paper is to show that the technique can be extended in order to solve transmission problems whereby an incident acoustic pressure wave impinges on a poroelastic material of finite dimension. For homogeneous and isotropic materials, the wave equation for the fluid phase and solid phase displacements are found to be decoupled thanks to the Helmholtz decomposition. This allows a systematic way for obtaining an analytic expression for the fundamental solution describing the wave displacement field in the material. The efficiency of the technique relies on choosing an appropriate set of fundamental solutions as well as properly imposing the transmission conditions at the air-porous interface.

In this paper, we address this issue showing results involving bidimensional scatterers of various shapes. In particular, it is shown that reliable error indicators can be used to assess the quality of the results. Comparisons with results computed using a mixed pressure-displacement finite element formulation illustrate the great advantage of this new technique both in terms of computational resources and mesh preparation.

Keywords: method of fundamental solutions, Biot's equations, poroelastic, porous material, scattering.
\end{abstract}




\section{Introduction}

Poroelastic materials are often used for their good sound absorbing capabilities in the middle and high frequency range. Typical applications can be found in the context of the transport noise reduction or to enhance the quality of the room acoustics. The description of wave propagation in porous media is not limited to audible acoustics as the Biot's model [1] was originally developed for geologic applications. Because of their inherent diphasic features and the strong contrasts that may exist between the solid and the fluid phases, the waves propagation modeling remains a difficult task often leading to heavy computational costs. In the context of Finite Element Method (FEM), some developments have been proposed using Lagrange or hierarchical finite elements $[2,3,4]$. Because of the disparity of scale, the so-called poroelastic elements have a slower convergence than purely elastic or fluid element [3]. To make the matter worst, Biot's equations are frequency dependent and large FEM system matrices have to be recalculated for each frequency. For homogeneous and isotropic materials, the Boundary Element Method (BEM) offers an alternative [5]. The method has the great advantage to reduce the entire problem to only unknowns on the boundaries. However, the system matrix is full and there is still the need to discretize the boundary domain as well as performing regular and singular integrations over each boundary elements.

In the past decade, several researchers have focused their work on meshless methods in order to avoid the time-consuming problem originated in mesh generation for complicated geometries. In this regard, the Method of Fundamental Solution (MFS) has been shown to be efficient for solving a large variety of physical problems as long as a fundamental solution of the underlying differential equation(s) is known. In particular the MFS is suitable for scattering problems by choosing appropriate fundamental solutions satisfying the radiation condition at infinity. The method shares the same advantages as BEM over domain discretization methods because there is no need to create a mesh over the entire domain. Furthermore, as no integration is needed, some numerical difficulties encountered with BEM are avoided. For comprehensive reviews on applications of the MFS one can refer to Fairweather et al. [6, 7].

In this work, we are interested in applying as well as assessing the MFS for the simulation of the $2 \mathrm{D}$ acoustic wave scattering by a poroelastic material. After presenting the MFS formulation in Section 2, the method's performances are measured against an academic problem for which exact analytical solutions are available. In particular, it is shown that reliable error indicators can be used to assess the quality of the results. The last section shows practical applications with comparisons with a FE model.

\section{Formulation of the method}

Consider a time-harmonic acoustic plane wave $\varphi_{0}^{\text {inc }}=\exp \mathrm{i} k_{0} \zeta \cdot \mathbf{x}$ (with the convention $\left.\mathrm{e}^{-\mathrm{i} \omega t}\right)$ in an unbounded exterior propagative domain $\Omega^{e}$ incident upon one (or more) poroelastic inclusion(s) denoted by $\Omega^{i}$ with boundary $\Gamma$ as shown in 


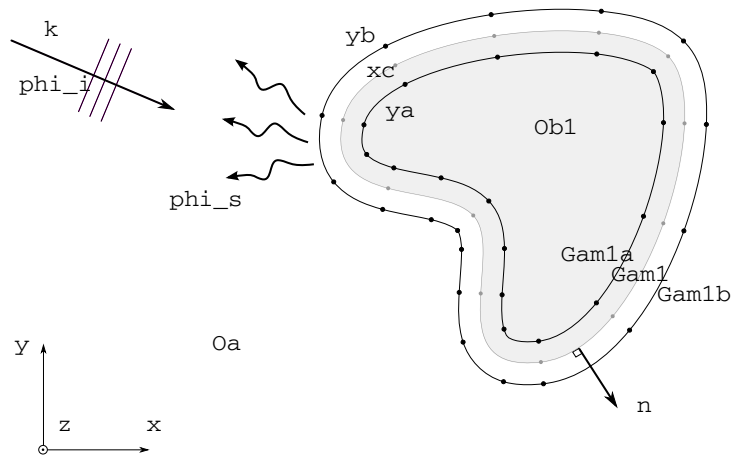

Figure 1: Scattering geometry.

Fig. 1. We call $\mathbf{n}$, the unit outward normal vector to $\Omega^{i}$. In the surrounding acoustic domain $\Omega^{e}$, the fluid is inviscid and the acoustic displacement potential $\varphi_{0}$ obeys the wave equation

$$
\Delta \varphi_{0}+k_{0}^{2} \varphi_{0}=0 .
$$

Here, $k_{0}=\omega / c_{0}$ is the classical wavenumber defined as the ratio of the angular frequency $\omega$ and the sound speed $c_{0}$. To express the transmission conditions at the interface $\Gamma$, it is convenient to introduce the particle displacement perturbation $\mathbf{w}=\nabla \varphi_{0}$. With this definition, the acoustic pressure is obtained from the linearized momentum equation as $p=\rho_{0} \omega^{2} \varphi_{0}$. In (1) it is natural to split the potential into an incident and a scattered part as $\varphi_{0}=\varphi_{0}^{\text {inc }}+\varphi_{0}^{\text {sc }}$ (and similarly for the pressure and displacement). Here we require the scattered field to satisfy the usual Sommerfeld radiation condition at infinity. In the poroelastic inclusions the acoustic waves propagation are described by Biot's model [1]. This latter is grounded on the superposition of a fluid phase and a solid phase which are coupled together and respectively described by the fluid phase displacement $\mathbf{U}$ and the solid phase displacement $\mathbf{u}$. For time-harmonic representation, we have the following coupled system [1]

$$
\begin{aligned}
& \nabla \cdot \boldsymbol{\sigma}^{s}+\omega^{2}\left(\rho_{11} \mathbf{u}+\rho_{12} \mathbf{U}\right)=0, \\
& \nabla \cdot \boldsymbol{\sigma}^{f}+\omega^{2}\left(\rho_{12} \mathbf{u}+\rho_{22} \mathbf{U}\right)=0 .
\end{aligned}
$$

Solid and fluid phase stress tensors are given by

$$
\begin{aligned}
& \boldsymbol{\sigma}^{s}=(A \nabla \cdot \mathbf{u}+Q \nabla \cdot \mathbf{U}) \mathbf{I}+2 N \boldsymbol{\varepsilon}^{s}, \\
& \boldsymbol{\sigma}^{f}=(Q \nabla \cdot \mathbf{u}+R \nabla \cdot \mathbf{U}) \mathbf{I}
\end{aligned}
$$

where $\varepsilon^{s}=1 / 2\left(\nabla \mathbf{u}+(\nabla \mathbf{u})^{\mathrm{t}}\right)$ is the usual strain tensor and $\mathbf{I}$ is the identity matrix. The total stress tensor $\boldsymbol{\sigma}^{t}$ is, by definition, the sum of $\boldsymbol{\sigma}^{f}$ and $\boldsymbol{\sigma}^{s}$. Biot's coefficients $A, N, Q, R$ are related to the material properties by the Allard-Johnson model. Their expressions can be found in the literature or in the reference textbook [8] 
as well as the other quantities introduced in this section. These quantities are all complex and frequency-dependent, $A$ and $N$ correspond to the Lamé coefficients, $R$ is the effective bulk modulus of the fluid phase and $Q$ indicates the coupling of the two phases volumic dilatation. The imaginary part of $A$ and $N$ includes the structural damping and, in $Q$ and $R$ this part includes the thermal dissipation. The imaginary parts of the effective density coefficients $\rho_{11}, \rho_{22}$ and $\rho_{12}$ take into account viscous damping. Now, the complete solution to the problem is found after applying the classical air-porous transmission conditions $[9,10]$ on the interface $\Gamma$, i.e.

$$
\begin{aligned}
p_{p}-p^{\mathrm{sc}} & =p^{\mathrm{inc}} \\
\phi \mathbf{U} \cdot \mathbf{n}+(1-\phi) \mathbf{u} \cdot \mathbf{n}-\mathbf{w}^{\mathrm{sc}} \cdot \mathbf{n} & =\mathbf{w}^{\mathrm{inc}} \cdot \mathbf{n}, \\
\boldsymbol{\sigma}^{t} \mathbf{n}+p^{\mathrm{sc}} \mathbf{n} & =-p^{\mathrm{inc}} \mathbf{n} .
\end{aligned}
$$

Here $\phi$ is the porosity and the pore pressure $p_{p}$ is obtained from the fluid phase tensor as $-\mathbf{I} \phi p_{p}=\boldsymbol{\sigma}^{f}$.

For homogeneous and isotropic materials, the wave equation for the fluid phase and solid phase displacements are found to be decoupled thanks to the Helmholtz decomposition. Both solid and fluid displacement fields are then written as

$$
\mathbf{u}=\nabla \varphi+\nabla \wedge\left(\varphi_{3} \cdot \mathbf{e}_{3}\right) \text { and } \mathbf{U}=\nabla \chi+\nabla \wedge\left(\Theta \cdot \mathbf{e}_{3}\right) .
$$

After equations decoupling, we have [8]: $\varphi=\varphi_{1}+\varphi_{2}$ and $\chi=\mu_{1} \varphi_{1}+\mu_{2} \varphi_{2}$ where

$$
\mu_{i}=\frac{P k_{i}^{2}-\omega^{2} \rho_{11}}{\omega^{2} \rho_{12}-Q k_{i}^{2}}, \quad i=1,2,
$$

are the waves amplitude ratios between the two phases in the porous material (here, $P=A+2 N$ ). Similarly, the potential $\Theta$ is simply obtained as $\Theta=\mu_{3} \varphi_{3}$ with $\mu_{3}=\rho_{12} / \rho_{22}$. Under this form, each potential $\varphi_{i}(i=1,2,3)$ fulfills the Helmholtz equation

$$
\Delta \varphi_{i}+k_{i}^{2} \varphi_{i}=0
$$

and the associated complex wavenumbers are given explicitly in [8]. Physically, there are two compressional waves associated with $\varphi_{1}, \varphi_{2}$ and one rotational (shear) wave associated with $\varphi_{3}$. They all propagate in the two phases and their relative contribution are given by the coefficients $\mu_{i}$. If such a decomposition holds in elastodynamics, the coexistence of two phases in the poroelastic media adds another fluid-born compressional wave which is not present is elastic solids.

The MFS theory starts by choosing an appropriate set of fundamental solutions for both propagative domains $\Omega^{e}$ and $\Omega^{i}$. In the acoustic domain, a natural choice is to pick these solutions using the well-known free field Green function, 
i.e. $G_{0}(\mathbf{x}, \mathbf{y})=\mathrm{i} / 4 H_{0}\left(k_{0}|\mathbf{x}-\mathbf{y}|\right)$. So we seek the scattered field as a distribution of monopole

$$
p^{\mathrm{sc}}(\mathbf{x})=\rho_{0} \omega^{2} \sum_{q_{0}=1}^{Q_{0}} A_{q_{0}}^{0} G_{0}\left(\mathbf{x}, \mathbf{y}_{q_{0}}^{0}\right),
$$

where source points $\mathbf{y}_{q_{0}}^{0}$ are chosen to be located on $\hat{\Gamma}_{0}$ in $\Omega^{i}$. The displacement vector is obtained by simply taking the gradient. To simulate the wave field in the inclusion, a possible choice is to use fundamental solutions for poroelastic media which explicit form can be found, for instance, in [11]. An easier option is to construct these solutions by simply expanding each potential in the form

$$
\varphi_{i}(\mathbf{x})=\sum_{q_{i}=1}^{Q_{i}} A_{q_{i}}^{i} G_{i}\left(\mathbf{x}, \mathbf{y}_{q_{i}}^{i}\right), \quad \text { for } i=1,2,3
$$

where $G_{i}(\mathbf{x}, \mathbf{y})=\mathrm{i} / 4 H_{0}\left(k_{i}|\mathbf{x}-\mathbf{y}|\right)$ is the fundamental solution for the Helmholtz equation. Here, $Q_{i}$ is the number of source points $\mathbf{y}_{q_{i}}^{i}$ associated with the $i^{\text {th }}$ potential and the $A_{q_{i}}^{i}$ 's are unknown amplitudes. These points are located on a fictitious boundary $\hat{\Gamma}_{i}$ in the exterior domain $\Omega^{e}$ as shown in Fig. 1 . The influence of the location of the source points on the quality of the solution will be discussed in the next section. Now, using these expansion in (6), we find the expression for the different physical quantities involved:

$$
\begin{aligned}
\phi p_{p}(\mathbf{x}) & =\sum_{i=1}^{2} k_{i}^{2}\left(Q+\mu_{i} R\right) \sum_{q_{i}=1}^{Q_{i}} A_{q_{i}}^{i} G_{i}\left(\mathbf{x}, \mathbf{y}_{q_{i}}^{i}\right) \\
\mathbf{u}(\mathbf{x}) & =\sum_{i=1}^{2} \sum_{q_{i}=1}^{Q_{i}} A_{q_{i}}^{i} \nabla G_{i}\left(\mathbf{x}, \mathbf{y}_{q_{i}}^{i}\right)+\sum_{q_{3}=1}^{Q_{3}} A_{q_{3}}^{3} \nabla_{\perp} G_{3}\left(\mathbf{x}, \mathbf{y}_{q_{3}}^{3}\right) \\
\mathbf{U}(\mathbf{x}) & =\sum_{i=1}^{2} \sum_{q_{i}=1}^{Q_{i}} A_{q_{i}}^{i} \mu_{i} \nabla G_{i}\left(\mathbf{x}, \mathbf{y}_{q_{i}}^{i}\right)+\sum_{q_{3}=1}^{Q_{3}} A_{q_{3}}^{3} \mu_{3} \nabla_{\perp} G_{3}\left(\mathbf{x}, \mathbf{y}_{q_{3}}^{3}\right)
\end{aligned}
$$

where $\nabla_{\perp} \equiv\left(\partial_{x_{2}},-\partial_{x_{1}}\right)^{\mathrm{t}}$ stands for the orthogonal gradient operator. From these expressions and using (4), we finally get the normal total stress tensor $\sigma^{t}$. The explicit forms for the tensor coefficients are too cumbersome to be inserted in this paper. Note that other type of solutions could have been considered (Green functions and/or their derivatives or plane waves for instance) and the present choice was mainly motivated for its simplicity. Furthermore, it has the great advantage of allowing us to specify independently the number of source points $Q_{i}$ for each kind of wave, this is not the case when using the Green function. In this respect, our method does not fall strictly into the category of the MPS since it does not rely on the Biot's equations displacements fundamental tensor. 
Now, given a set of $N_{\text {col }}$ collocation points $\mathbf{x}_{l}\left(l=1, \ldots, N_{\mathrm{col}}\right)$. on $\Gamma$, substituting (11) in the transmission conditions (5) at each collocation point yields three linear system of the form

$$
\mathbf{M}_{\alpha} \mathbf{A}=\mathbf{F}_{\alpha}^{\mathrm{inc}}, \quad \alpha=\mathrm{a}, \mathrm{b}, \mathrm{c} .
$$

Here, subscript $\alpha$ refers to the type of condition involved (there is one system for each condition (5a), (5b), (5c)). The right hand side vector $\mathbf{F}_{\alpha}^{\text {inc }}$ stems from the incident wave (pressure and displacement). The unknown vector $\mathbf{A}$ contains the amplitudes of all sources. Since the acoustic displacement is expected to behave like $|\mathbf{w}| \sim k_{0} \phi_{0}$, it is judicious to rescale the cinematic conditions (5b) by multiplying the associated lines by $\rho_{0} c_{0} \omega$. This yields the matrix system

$$
\mathbf{M A}=\mathbf{F}^{\text {inc }} .
$$

In this work, early results showed that it is preferable for reasons of stability to consider more collocation points than the number of unknowns $N_{\text {dof }}=\sum_{i=0}^{3} Q_{i}$. To be more specific, it was found that taking $N_{\text {col }}=2 N_{\text {dof }}$ guarantees that results have converged, i.e. the numerical solution becomes insensitive to the number of collocation points. In the following, all calculations are performed using this ratio. Note that when dealing with 'nice' shaped inclusions (the circular scatterer for instance), the MFS has been observed to perform a bit better with interpolation schemes (i.e. $\left.N_{\text {col }}=N_{\text {dof }}\right)$ than least-square schemes $\left(N_{\text {col }}>N_{\text {dof }}\right)$. However, it was found preferable for the sake of robustness and generality to favor the second approach.

After multiplying by the hermitian transpose $\mathbf{M}^{\dagger}$ we finally get

$$
\mathbf{M}^{\dagger} \mathbf{M A}=\mathbf{M}^{\dagger} \mathbf{F}^{\text {inc }} \text {. }
$$

The two advantages for doing this operation are (i) having to deal with a hermitian square system and (ii) that a simple error estimator is available. Indeed, coming back to the original problem (12), we can define the a posteriori error estimator for each conditions at the interface $(\alpha=\mathrm{a}, \mathrm{b}, \mathrm{c})$. So we put

$$
\mathcal{E}_{\alpha}=100 \frac{\left\|\mathbf{M}_{\alpha} \mathbf{A}-\mathbf{F}_{\alpha}^{\text {inc }}\right\|_{2}}{\left\|\mathbf{F}_{\alpha}^{\text {inc }}\right\|_{2}},
$$

where $\mathbf{A}$ is solution of (14).

As for the choice for the source points positions, we follow Alves [12] and take these points along the discrete normal direction, so we put $\mathbf{y}_{q_{i}}^{i}=\hat{\mathbf{y}}_{q_{i}}^{i}+s^{i} \tilde{\mathbf{n}}_{q_{i}}^{i}$ where points $\hat{\mathbf{y}}_{q_{i}}^{i}\left(q_{i}=1, \ldots, Q_{i}\right.$ and $\left.i=0, \ldots, 4\right)$ are distributed on $\Gamma$ and $\tilde{\mathbf{n}}_{q_{i}}^{i}$ is the approximate normal vector defined by

$$
\tilde{\mathbf{n}}_{q_{i}}^{i}=\frac{\left(\hat{\mathbf{y}}_{q_{i}+1}^{i}-\hat{\mathbf{y}}_{q_{i}-1}^{i}\right)^{\perp}}{\max _{q_{i}}\left|\hat{\mathbf{y}}_{q_{i}+1}^{i}-\hat{\mathbf{y}}_{q_{i}-1}^{i}\right|} .
$$

Here, symbol $\perp$ signifies that we take the orthogonal vector pointing outward. The normalization is chosen here for pure convenience to make sure that the normal 
vectors amplitudes never exceed the unity and that coefficients $s^{i}$ correspond to the farthest distance from the interface $\Gamma$.

\section{Validations for the circular-shaped inclusion}

We shall validate and assess the efficiency of the method on the specific case of the scattering of a horizontal acoustic plane wave by a circular-shaped poroelastic inclusion. In polar co-ordinates, the inner and outer wave fields can be represented by separable solutions. Each potential is given by infinite series which are well behaved allowing us to produce very accurate results without deterioration at high frequency. The derivation of the exact form for these solutions is quite lengthy and this will not be inserted in the present paper. For a proper assessment of the method's performance, it is convenient to define the relative error on the boundary for each physical quantity (call it $X$ ):

$$
\hat{\mathcal{E}}(X)=100 \frac{\|\mathbf{X}-\hat{\mathbf{X}}\|_{2}}{\|\hat{\mathbf{X}}\|_{2}},
$$

where $X$ represents either the acoustic pressure, the fluid phase or solid phase normal displacement or the normal stress at the interface and $\mathbf{X}$ is the associated vector containing the value of $X$ at the collocation points. Here, $\hat{\mathbf{X}}$ stands for a reference solution vector, either obtained analytically in the present case or numerically computed with another method when analytical solutions are not available. Now, the aim of this section is to identify the effects of the main parameters of the problem, namely the sources locations and the number of sources. For this latter, we are still left with the problem of finding a quasi-optimal relationship between the $Q_{i}$ 's. Through extremely intensive calculations, not shown here, we found that choosing the same number for each wave type was probably the best option offering the best trade-off between accuracy, conditioning and simplicity. This greatly simplifies the analysis as we can now put $Q=Q_{i}$ for $i=0, \ldots, 4$ and perform the analysis with a single parameter $Q$. Similarly, we take the points $\hat{\mathbf{y}}_{q_{i}}^{i}$ all equal and put $s^{i}=s$ with $s^{0}=-s$. Note this choice seems to be against results given in [13] where it is advocated that the best option, when using plane waves, is precisely not to take the same number of wave directions for each type of waves (that is shear and pressure wave types in the elasticity case) and this is particularly relevant when the ratio between wavenumbers is large. The reasons for this probably lie in that, plane waves and singular sources behave differently in terms of their approximation properties and it would be interesting to explore this further via numerical experiments in the spirit of [14] for instance.

For the sake of illustration we chose a polymer foam (XFM) commonly used in the transport industry. The materials properties are reported in Tab. 1. To give an idea of the complex wavenumbers for the wave potentials in the material, we find that $k_{1} \approx 17.6+1.21 \mathrm{i}, k_{2} \approx 21.4+14.1 \mathrm{i}$ and $k_{3} \approx 38.9+2.1 \mathrm{i}$ (these values corresponds to the frequency $500 \mathrm{~Hz}$ ). The corresponding acoustic wavenumber is 
188 Boundary Elements and Other Mesh Reduction Methods XXXII

Table 1: Materials properties for the XFM foam. All quantities reported are defined in [9].

\begin{tabular}{cccccccc}
\hline \hline$\phi$ & $\sigma$ & $\alpha_{\text {inf }}$ & $\Lambda$ & $\Lambda^{\prime}$ & $\rho_{1}$ & $N$ & $\nu$ \\
\hline 0.98 & 13.5 & 1.7 & 80 & 160 & 30 & $200(1-0.05 i)$ & 0.35 \\
\hline \hline
\end{tabular}

$k_{0} \approx 9.2$. On Fig. 2 (a) are shown the influence of the source locations as well as the number of sources (i.e. $Q$ ) on the normal solid displacement error $u_{n}=\mathbf{u} \cdot \mathbf{n}$, i.e. $\hat{\mathcal{E}}\left(u_{n}\right)$ and the a posteriori error $\mathcal{E}_{\mathrm{b}}$. The evolution of the conditioning (in the 2-norm) of the associated system is also shown (Fig. 2 (b)). Before we comment on these results, we should point out that we chose to measure the normal displacement error rather than the pressure error for the simple reason that it was observed, that a small error on the displacement normally guarantees a smaller error on the pressure (though the opposite is not true). The convergence curves together with the conditioning curves typically illustrate the MFS paradigm which many authors have already observed and discussed: ill conditioned systems lead to accurate results. In the present situation, it is concluded that the further away from the boundary the source points are (i.e. the higher $s$ ), the better the results as long as the conditioning of the system does not exceed a certain value above which results are likely to be corrupted by round off errors. This value, say cond ${ }_{2} \approx 10^{16}$, is in line with standard double precision arithmetic. Note that, in our algorithm, matrices are inverted using the pinv function from MATLAB so that for very illconditioned matrices $\left(\operatorname{cond}_{2}>10^{16}\right)$ the SVD solver is automatically used with some thresholding in order to dampen the effects of the round-off errors. In this
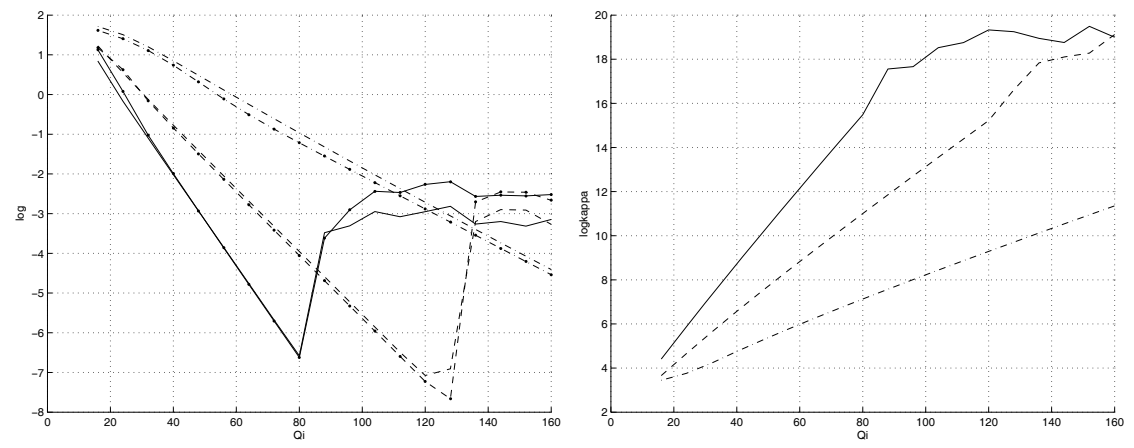

Figure 2: Effects of the sources location on the error and the conditioning $(--s=0.1 a,--s=0.2 a,-s=0.3 a)$. (a): error on the solid phase normal displacement for the XFM foam at $1500 \mathrm{~Hz}$; line with markers - refers to $\hat{\mathcal{E}}\left(u_{n}\right)$ error and the simple line to the a posteriori error $\mathcal{E}_{\mathrm{b}}$. (b): conditioning (2-norm) of $\mathbf{M}^{\dagger} \mathbf{M}$. 
matter, we think that better results could perhaps be made by properly filtering the small singular values. Another approach would be to apply the Tikhonov regularization in order to speed up the computation.

In fact, the most striking feature of these convergence curves is the almost perfect adequation between the true error $\hat{\mathcal{E}}\left(u_{n}\right)$ and the its algebraic counterpart, the a posteriori error $\mathcal{E}_{\mathrm{b}}$ and through lots of numerical experiments, we always observe very good correlations. The mathematical reasons for this goes beyond the scope of this paper but we believe that in most cases the algebraic a posteriori errors $\mathcal{E}_{\alpha}$ should serve as good error indicators especially when reference solutions are not available.

\section{Numerical examples and concluding remarks}

This section describes two examples illustrating the efficiency of the MFS. The first shows the MFS performances for inclusions presenting geometrical singularities such as corners and in the second example, we tackle multiple scattering problems with non-convex shaped objects. Because there are no analytical solutions to these problems, all reference solutions are computed using Finite Element models. These computations are carried using Lagrange quadratic finite element in both fluid and poroelastic domains. The $\left(\mathbf{u}, p_{p}\right)$ formulation of Biot's equations $[2,9]$ is used and the non reflecting boundary conditions are implemented using the Bermudez's perfectly matched layer formulation [15] (PML). As for the accuracy of these FE reference solutions, numerical tests carried out on the circular shaped scattering problem shows that these results are reliable up to around 1 percent of error on the solid phase normal displacement which is acceptable if engineering accuracy is sought. The first example concerns that of a square-shaped poroelastic inclusion of side length $a=0.2 \mathrm{~m}$. The incident plane wave is horizontal travelling in the $\zeta=(1,0)$ direction and the frequency is $1500 \mathrm{~Hz}$. In this scenario, the boundary curve is not analytic and source points around the square are placed along the normal to the boundary avoiding the corners, see Fig. 3 (a).
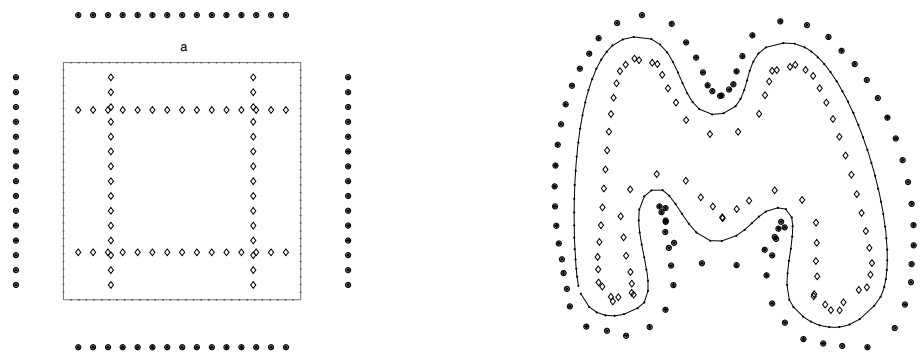

Figure 3: (a) Square-shaped inclusion. (b) The ' $M$ ' shaped scatterer. Here symbols ,$+ \times, \circ$ indicate the location of the source points of $\Omega^{e}$ and $\diamond$ those of $\Omega^{i}$. The collocations point on $\Gamma$ are identified by the $\cdot$ markers. 

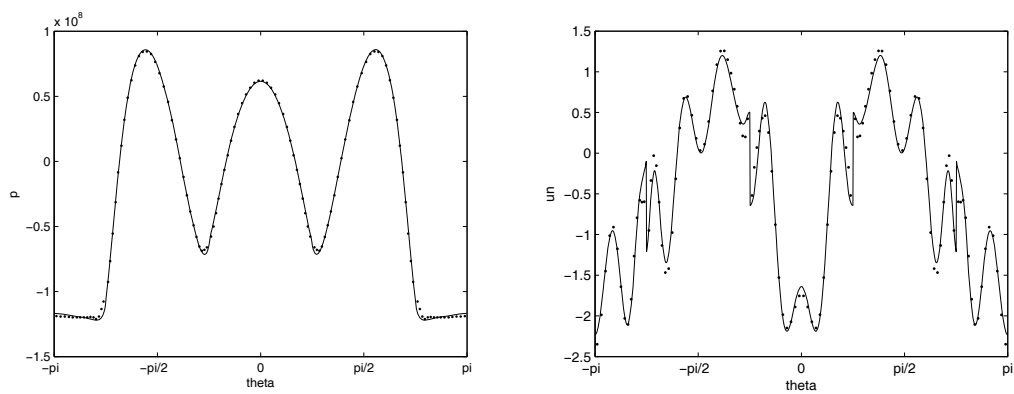

Figure 4: (a): Real part of the pressure field on $\Gamma$ at $1500 \mathrm{~Hz}$ for the square-shaped inclusion; solid line: FEM solution and dots refers to the MFS solution. (b): Real part of solid phase normal displacement.

In this respect, we found that putting more sources in the vicinity of the corners did not show any improvement. Comparisons between the MFS and the FE results are conveniently shown in Fig. 4. Here, the abscissae $\theta$ refers the usual polar coordinate of the collocation points on the boundary (the square is centered at the origin). As expected, the discrepancies are maximum near the corners and this is particularly noticeable for the displacement curve. The associated global errors are in good agreements as we found $\mathcal{E}_{\mathrm{b}}=4.42 \%$ with $\hat{\mathcal{E}}\left(u_{n}\right)=1.88 \%$ at 500 $\mathrm{Hz}$ and $\mathcal{E}_{\mathrm{b}}=4.95 \%$ with $\hat{\mathcal{E}}\left(u_{n}\right)=4.31 \%$ at $1500 \mathrm{~Hz}$. Here we took $Q=60$ $\left(N_{\text {dof }}=240\right)$.

The last numerical experiment concerns a multiple scattering problem with nonconvex objects having the shape of the three letters ' $M$ ', ' $F$ ' and ' $S$ '. The boundaries have been drawn using a graphical software and interpolated with Bezier curves. Fig. 3 (b) shows the location of the source points as well as the collocation points on the ' $M$ ' letter. The example considered here is that of an incident plane wave travelling in the $\zeta=(\cos \pi / 4,-\sin \pi / 4)$ direction and the frequency is $6000 \mathrm{~Hz}$. In our calculation, we took $Q=80$ for each scatterer; this yields $N_{\text {dof }}=3 * 4 * Q=960$ variables. The a posteriori errors for the pressure and the displacement are $\mathcal{E}_{\mathrm{a}}=1.78 \%$ and $\mathcal{E}_{\mathrm{b}}=2.77 \%$. The total pressure in the acoustic domain and in the porous inclusions is shown in Fig. 5. The absorbing properties of the porous material can be visually identified. Note that the CPU time needed for computing the matrix coefficients and inverting the system does not exceed 20 s on a single PC (Intel Core 2 Duo T7100 @ $1.80 \mathrm{GHz}$ ) and the use of the FEM would be far more demanding in order to achieve the same level of accuracy.

Through these examples, we showed the great advantage of the MFS both in terms of computational resources and mesh preparation. When dealing with scatterers presenting geometrical singularities such as corners, comparisons with FE computations showed that few percent errors results can still be achieved which is acceptable if engineering accuracy is sought. It is believed that this could have a positive impact for solving a wide range of noise control problems ranging from 


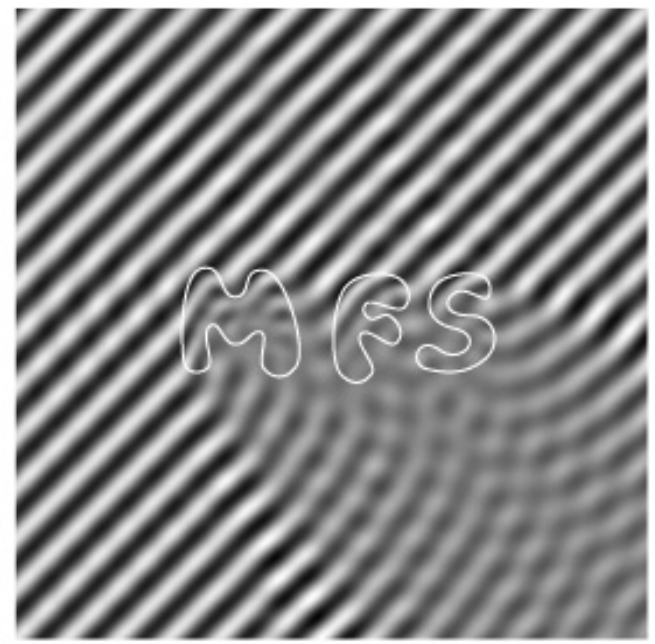

Figure 5: Real part of the total pressure in the acoustic domain and in the porous inclusions.

multiple scattering to shape foam optimization. Work is ongoing by the authors to extend the method to $3 \mathrm{D}$ configurations.

\section{References}

[1] Biot, M.A., Theory of propagation of elastic waves in a fluid-saturated porous solid. I. low-frequency range. II. higher frequency range. J Acoust Soc Am, 28(2), pp. 168-191, 1956.

[2] Atalla, N., Hamdi, M.A. \& Panneton, R., Enhanced weak integral formulation for the mixed $(u, p)$ poroelastic equations. J Acoust Soc Am, 109(6), pp. 3065-3068, 2001.

[3] Rigobert, S., Atalla, N. \& Sgard, F., Investigation of the convergence of the mixed displacement-pressure formulation for three-dimensional poroelastic materials using hierarchical elements. J Acoust Soc Am, 114(5), pp. $2607-$ 2617, 2003.

[4] Horlin, N.E., Nordstron, M. \& Goransson, P., A 3-D hierarchical fe formulation of biot's equations for elasto-acoustic modeling of porous media. $J$ Sound Vib, 245(4), pp. 633-652, 2001.

[5] Tanneau, O., Lamary, P. \& Chevalier, Y., A boundary element method for porous media. J Acoust Soc Am, 20(3), pp. 1239-1251, 2006.

[6] Fairweather, G., Karageorghis, A. \& Martin, P.A., The method of fundamental solutions for scattering and radiation problems. Engng Anal Bound Elem, 27, pp. 759-769, 2003. 
[7] Fairweather, G. \& Karageorghis, A., The method of fundamental solutions for elliptic boundary value problems. Adv Comput Math, 9(1-2), pp. 69-95, 1998.

[8] Allard, J.F., Propagation of Sound in Porous Media: Modeling Sound Absorbing Materials. Chapman \& Hall, 1993.

[9] Debergue, P., Panneton, R. \& Atalla, N., Boundary conditions for the weak formulation of the mixed $(u, p)$ poroelasticity problem. J Acoust Soc Am, 106(5), pp. 2393-2390, 1999.

[10] Lovera, O.L., Boundary conditions for a fluid-saturated porous solid. Geophysics, 52(2), pp. 174-178, 1987.

[11] Bonnet, G., Basic singular solutions for a poroelastic medium in the dynamic range. J Acoust Soc Am, 82(5), pp. 1758-1762, 1987.

[12] Alves, C.J.S., On the choice of source points in the method of fundamental solutions. Engng Anal Bound Elem, 33, pp. 1348-1361, 2009.

[13] Perrey-Debain, E., Plane wave decomposition in the unit disc: Convergence estimates and computational aspects. J Comput Appl Math, 193(1), pp. 140 156, 2006.

[14] Alves, C.J.S. \& Valtchev, S.S., Numerical comparison of two meshfree methods for acoustic wave scattering. Engng Anal Bound Elem, 29, pp. 371-382, 2005.

[15] Bermudez, A., Hervella-Nieto, L., Prieto, A. \& Rodriguez, R., An optimal perfectly matched layer with unbounded absorbing function for timeharmonic acoustic scattering problems. J Comput Phy, 223, pp. 469-488, 2007. 